\title{
A separate design principle for priority-aware packet collection in industrial cyber-physical systems
}

\author{
Feilong Lin ${ }^{1,2}$, Cailian Chen ${ }^{1,2^{*}}$, Qimin Xu ${ }^{1,2}$, Cunqing Hua ${ }^{3}$ and Xinping Guan ${ }^{1,2}$
}

\begin{abstract}
Industrial cyber-physical systems (ICPS) bridge the physical factory floor and the cyber computational space by leveraging the emerging techniques, such as wireless sensor networks for ubiquitous connection and perception, where sensors are deployed to monitor and collect industrial data according to different timeliness requirements. Priority-aware schemes are often used to tackle the differentiated data packet collection. However, it is challenging to coordinate the multichannel access while meeting the priority-aware transmission requirement in ICPS. In this paper, we propose a separate design principle (SDP) for priority-aware packet collection. In SDP, the transmission of each priority class of sensors is separately scheduled by a multichannel superframe which fully uses the available channels. As a result, each time slot on each channel is repeatedly scheduled to sensors of different priorities by different superframes. Then, a priority-aware transmission mechanism is devised to coordinate the transmissions of different sensors in the predefined priority order. Simulation results show that for four priorities, SDP achieves as low as 16, 18, 23 , and $36 \%$ of the mean packet transmission delay of non-overlap TDMA scheduling for each priority class, respectively. Moreover, SDP greatly outperforms IEEE 802.15.4e protocol for packet collection with two priorities. We also demonstrate the feasibility of SDP based on the implementation of software-defined radios.
\end{abstract}

Keywords: Industrial cyber-physical systems, Priority-aware packet collection, Multichannel scheduling, Separation design principle

\section{Introduction}

Cyber-physical systems (CPS) are an integrated infrastructure that involves sensing, computation, communications, and control $[1,2]$. Manufacturing industry integrated with CSP, aka industrial cyber-physical systems (ICPS), bridges the physical factory floor and the cyber computational space by leveraging the emerging techniques, such as wireless sensor networks (WSNs) for ubiquitous connection and perception [3]. ICPS have been considered as the landmark in the development of Industry 4.0, the next generation of manufacturing industry. It is reported by GE that about $46 \%$ of the global economy

\footnotetext{
*Correspondence: cailianchen@sjtu.edu.cn

1 Department of Automation, Shanghai Jiao Tong University, 800, Dongchuan Rd., Minhang District, 200240 Shanghai, People's Republic of China

2 Key Laboratory of Systems Control and Information Processing, Ministry of

Education of China, 800, Dongchuan Rd., Minhang District, 200240 Shanghai, People's Republic of China
}

Full list of author information is available at the end of the article or \$32.3 trillion in global output can benefit from ICPS [4].

In ICPS scenario, sensors are deployed and various monitoring data are to be collected according to the different timeliness requirements. For example, industrial process monitoring data transmission for online control can only tolerate the latency no more than tens of milliseconds [5]. Thus, they require higher transmission priority than other monitoring data which may allow long latency, such as the machine health monitoring data. Priorityaware schemes are often used to tackle the differentiated data transmission. However, it is still challenging to coordinate the multichannel access while meeting the priorityaware transmission requirement due to the complexity of collision avoidance and transmission prioritization.

Since the IEEE Std 802.15.4 [6] was released, which defines the MAC and PHY specifications in ISM (industrial, scientific, and medical) band, several standardized

\section{Springer}

(c) 2016 Lin et al. Open Access This article is distributed under the terms of the Creative Commons Attribution 4.0 International License (http://creativecommons.org/licenses/by/4.0/), which permits unrestricted use, distribution, and reproduction in any medium, provided you give appropriate credit to the original author(s) and the source, provide a link to the Creative Commons license, and indicate if changes were made. 
protocols have been successively released, such as Zigbee [7], WirelessHART [8], ISA100.11a [9], and WIA-PA [10]. Zigbee, utilizing CSMA/CA (carrier sense multiple access/collision avoidance) mechanism, provides the capability of self-organization and scalability. However, it is not efficient enough for large-scale networks. As shown in $[1,11]$, when more than 20 nodes in the network, the packet delivery ratio falls below $40 \%$ and the packet transmission delay exceeds $100 \mathrm{~ms}$. Hence, CSMA/CA is not suitable to serve the ICPS with a large number of devices and strict timeliness requirement. As an alternative solution, TDMA (time-division multiple access), which achieves the deterministic and predictable transmission, is accepted as the main scheduling mechanism by WirelessHART, ISA100.11a, and WIA-PA. By this reservation-based scheduling approach, these three protocols have specified four transmission priorities for different data, i.e., command, process data, normal, and alarm from the highest priority to the lowest. However, TDMA also faces two folds of deficiencies. Firstly, it cannot provide timely access for the transmission requests, especially in the random event-driven scenario. Second, since each slot is exclusively scheduled to one sensor, it may lead to waste if the sensor does not have data to transmit.

In this paper, we propose a flexible and efficient TDMA scheduling mechanism for priority-aware packet collection in ICPS. Consider the industrial WSN consisting of multiple classes of sensors, where each class of sensors belongs to different transmission priorities. The data packets from higher priority sensors have transmission superiority over the packets from lower priority sensors. We introduce a separate design principle (SDP) based on the multichannel superframe used in IEEE 802.15.4e [12]. The main idea of SDP is to separately design multichannel superframe for each class of sensors by fully utilizing the channels in the network. As a result, each time slot on each channel is repeatedly scheduled to sensors of different priorities by different superframes. Then, a priority coordinator is devised to manage the transmission according to the priority order. At the beginning of each slot, certain sub-slots are reserved for sensors to indicate their transmission priority. By checking the priority indicators, the lower priority sensors can opportunistically utilize the unused slots which have been scheduled to higher priority sensors. We preliminarily reported the SDP with two priority classes in [13]. In this paper, we extend the SDP to multipriority packet collection scenario and present comprehensive theoretical analysis for SDP. The main contributions of this work are summarized as follows:

- A separate design principle is proposed for multichannel scheduling in priority-aware packet collection. SDP not only guarantees the transmission priority of different sensors but also decreases the transmission delay of the lower priority sensors by allowing them to reuse the slots scheduled to higher priority sensors opportunistically.

- Noting that the periodic transmission benefits the decrease of packet transmission delay, a greedy multichannel superframe determination (GMSD) algorithm is devised to optimize the multichannel superframe design.

- The lower bound of mean packet transmission delay for each class of sensors is derived. It is also proved that the SDP-based scheduling obtains lower mean waiting delay than non-overlap TDMA scheduling.

The remainder of this paper is organized as follows: Section 2 presents a simple review of the related works. Priority-aware multichannel scheduling problem is described in Section 3. The main results of this work are derived in Section 4. Section 5 shows the performance analysis for the proposed SDP. Finally, simulations and experiments are conducted in Section 6 and the paper is concluded in Section 7.

\section{Related works}

Priority-aware packet collection is a fundamental technique to coordinate the sensors' data packet transmission with different timeliness requirements in ICPS. IEEE Standard 802.15.4, extensively applied in industrial applications, has provided the preliminary solution for packet transmission with different delay constraints [6]. By reserving the guaranteed time slots (GTSs) in contentionfree period (CFP), the high-priority data packets can be transmitted in a collision-free way. In contrast, the lowpriority data packets are committed to access to channel by CSMA-CA approach in contention access period (CAP). However, for large-scale network, the transmission efficiency is very low [11]. Based on IEEE 802.15.4 protocol, an adaptive strategy is proposed in [14] to make the tradeoff between CFP and CAP, thus to deal with the dynamics of high- and low-priority traffics. Compared to reserved transmission for high-priority packets, the authors in [15] propose to transmit high-priority packets in event-driven manner while the low-priority packets in TDMA manner. By allowing high-priority packet to hijack the transmission chance of low-priority packet, this approach guarantees the transmission superiority of high-priority packets. It works well in the scenario where the high-priority packet rate is very low, and no collision would happen among the transmissions of the highpriority packets. The authors in $[16,17]$ do not consider the absolute packet transmission priority; instead, they propose a proportional delay model and a transmission scheduler based on queueing information. As a result, 
the mean transmission delays of different data traffics approach a proportional fashion. In [18-20], the packet transmission priority from the perspective of remainder hops to the destination node is considered, and then, the dynamic scheduling methods are proposed to minimize the end-to-end delay.

The studies in above literatures are specified on single channel, which necessitate the multichannel access coordination to extend to multichannel industrial WSNs. To exploit the multichannel diversity, the authors in [21] propose a multichannel superframe scheduling mechanism for cluster-tree topology network based on IEEE 802.15.4 protocol. It allocates each cluster with one orthogonal channel to avoid inter-cluster interference. The superframe is still defined on single channel. A fixed priority packet transmission method based on WirelessHART is proposed in [22] for multichannel multihop networks. To meet the delay constraint, each sensor determines the transmission order of packets with the consideration of their remaining hops to the destination. Multiple channels are used to support parallel transmissions over multiple routing paths to reduce the end-to-end delay of packet delivery. In [23], the authors considered the multipriority multichannel access in cognitive radio environment. Similar to [15], high-priority traffics are allowed to preempt transmission chances of low-priority traffics. The authors in [24] proposed a distributed priority-aware multichannel access scheme based on weighted congestion game, where higher priority traffics are assigned with larger weight coefficients, thus to acquire more spectrum resource.

For priority-aware packet collection in industrial field, the access point (AP) cannot easily acquire the timely queueing states of sensors without efficient channel access mechanism. Hence, the TDMA scheduling, a reservationbased mechanism, is an appropriate choice [5]. We aim to reduce the packet transmission delay of each priority class of sensors by designing separate multichannel superframes and priority-aware transmission coordination mechanism. Further, it is worth to note that for each sensor, the evenness of the distribution of scheduled slots takes effect on the packet collection delay. To the best of our knowledge, this issue has not been considered in the existing works. In this paper, we explore the effect of the evenness on transmission delay and develop an algorithm to optimize the multichannel superframe design.

\section{Problem description}

\subsection{Network models}

Consider a star topology network in the industrial field. The sensors are deployed to monitor the industrial process and one access point (AP) is appointed to collect the data packets from the sensors. Due to different timeliness requirements, the sensors are classified to $C$ classes with different transmission priorities. Suppose that each class $c$ consists of $N_{c}$ sensors and the sensors in class $c$ are assigned with higher transmission priority over any class $c^{\prime}>c$. Similar to some existing studies [15, 23], the data packet flows generated by each class of sensors follow independent Poisson processes with packet generating rate $\left\{\lambda_{n, c} \mid n=1, \ldots, N_{c}, c=1, \ldots, C\right\}$. Suppose that the rates are slowly time-varying. For example, they keep invariable during the production of one batch or one order. The packet size is fixed and normalized to 1 .

Suppose that the network operates on $L$ channels. Time is slotted and the network is synchronized. During each time slot, one packet can be transmitted on each channel. The packet transmission of each sensor follows first-come first-served manner. Each sensor only transmits on a single channel in a slot, but can do per-slot channel hopping if required. AP supports multichannel transmission, which covers all $L$ channels.

To guarantee the transmission schedulability of the network, the average packet arrival rate in the network should be less than $L$. Accordingly, the network traffic rate must satisfy $\sum_{c=1}^{C} \sum_{n=1}^{N_{c}} \lambda_{n, c}<L$.

\subsection{Priority-aware multichannel scheduling problem}

The purpose of this paper is not only to coordinate the multipriority packet collection in multichannel networks but also to reduce the packet transmission delay for each priority class of sensors. To this end, the following two sub-problems are considered.

\subsubsection{Optimal multichannel superframe design}

It is observed that the TDMA-based multichannel scheduling would affect the packet transmission delay. The evenness of the distribution of scheduled time slots for each sensor, in particular the second moment of scheduling intervals, affects the mean packet transmission delay, which will be proved in Section 4.1.2. Therefore, the delay optimization should be considered in the multichannel superframe design.

\subsubsection{Priority-aware transmission coordination}

In order to meet the differentiated packet collection requirements, a priority-aware transmission coordination mechanism is needed. This coordination mechanism manages the transmission of different sensors in the predefined priority order. Besides the resolution of transmission priority, the transmission delay of each priority class of sensors can be further improved by devising proper transmission coordination mechanism.

\section{Main results}

This section first presents the optimal multichannel superframe design. Based on multichannel superframe 
design, the SDP for priority-aware packet collection is then derived.

\subsection{Multichannel superframe design}

Recently, IEEE 802.15.4e [12] has been released, in which the multichannel superframe is defined by the deterministic and synchronous multichannel extension (DSME) mechanism. To solve the first problem presented in Section 3.2.1, we use TDMA-based multichannel scheduling for the superframe design, such as DSME-GTS allocation in CFP in IEEE $802.15 .4 \mathrm{e}$ protocol. The CAP can be used to broadcast beacon frame to deliver the scheduling information to the sensors, and which can be set to a small period, e.g., one time slot. This work mainly considers the transmission scheduling in CFP and will not refer to the phase of the transmission in CAP.

\subsubsection{TDMA-based multichannel superframe design}

The basic idea of SDP is to separately design a multichannel superframe for each priority class of sensors. Without loss of generality, consider the multichannel supframe design for a general priority class with $N$ sensors (the priority order $c$ is omitted in this section). The packet arrivals of sensors are Possion-distributed with rates $\left\{\lambda_{n} \mid n=\right.$ $1, \ldots, N\}$. Denote $\mathrm{A}$ as the multichannel superframe on $L$ channels over a period of $T$ slots, which can be represented by a $L \times T$ matrix. Each element $a_{i, j}$ is one resource block of the $j$ th slot on the $i$ th channel. All the resource blocks of $\mathbf{A}$ are allocated to sensors in a proportional way. By taking the packet rate of each sensor as the proportional coefficient, the portion of resource allocated to each sensor is

$$
R_{n}=\frac{\lambda_{n}}{U} L T
$$

where $U=\sum_{n=1}^{N} \lambda_{n}$ is the overall packet rates of the sensors. In implementation, $R_{n}$ is set to be an integer number, and $\sum_{n=1}^{N} R_{n}=L T$.

In this context, the multichannel superframe design problem is to allocate the $L T$ resource blocks to $N$ sensors properly. For simplicity, we assign an integer to each $a_{i, j}$ to represent the resource block allocation in $\mathbf{A}$, e.g., $a_{i, j}=n$ means that $j$ th slot on $i$ th channel is allocated to sensor $n$. In practice, the whole superframe information is not necessary for each sensor. To save the storage resource, each sensor only needs to store its transmission schedule, denoted by $\mathbb{A}_{n}=\left\{(i, j) \mid a_{i, j}=n\right\}$. Note that $\mathbb{A}_{n}$ is a twodimensional array. Obviously, the length of the array $\mathbb{A}_{n}$ is $R_{n}$. Since no more than one channel can be allocated to a single channel sensor at each time slot, $\mathbb{A}_{n}$ has a strictly increasing order with respect to time slot index $j$.

\subsubsection{Mean packet transmission delay}

Denote $\left\{t_{n, k} \mid k=1, \cdots, R_{n}\right\}$ as the time slot indexes in transmission schedule $\mathbb{A}_{n}$. Then, with regard to the superframe $\mathbf{A}$, the scheduling interval for sensor $n$ is defined as

$$
s_{n, k}= \begin{cases}t_{n, k+1}-t_{n, k}, & k=1, \ldots, R_{n}-1, \\ T-t_{n, k+1}+t_{n, k}, & k=R_{n} .\end{cases}
$$

When the sensor delivers data packets according to the superframe periodically, the first and second moments of $\left\{s_{n, k}\right\}$ are

$$
\begin{aligned}
& \bar{s}_{n}=\frac{T}{R_{n}}, \\
& s_{n}^{(2)}=\frac{1}{R_{n}} \sum_{k=1}^{R_{n}}\left(s_{n, k}\right)^{2} .
\end{aligned}
$$

The packet transmission of each sensor can be modeled as a queueing process with Poisson arrival. Then, the packet transmission follows as the general distribution with the mean transmission interval $\bar{s}_{n}$ and the second moment $s_{n}^{(2)}$. According to M/G/1 queueing model [25], the mean waiting time of sensor $n$ can be obtained as follows according to Pollaczek-Khintchine formula:

$$
W_{n}=\frac{\lambda_{n} s_{n}^{(2)}}{2\left(1-\lambda_{n} \bar{s}_{n}\right)} .
$$

To emphasize the influence of scheduling interval on the mean waiting delay, we have the following lemma.

Lemma 1. For Poisson-distributed packet arrival, a lower second moment of scheduling interval achieves a lower mean waiting delay. The lowest mean waiting delay is achieved by periodic scheduling, i.e.,

$$
W_{n}^{L B}=\frac{\lambda_{n} T^{2}}{2 R_{n}^{2}-2 \lambda_{n} T R_{n}},
$$

and the lower bound of mean packet transmission delay (including the waiting slots and the transmission slot) of sensor $n$ is

$$
D_{n}^{L B}=W_{n}^{L B}+1 .
$$

Proof. From (5), a smaller $s_{n}^{(2)}$ results in smaller $W_{n}$. Note that the minimum $s_{n}^{(2)}$ is achieved when all resource blocks assigned to the sensor $n$ are equally distributed in time-dimension, i.e., $s_{n}^{(2)}=\left(\frac{T}{R_{n}}\right)^{2}$. Hence, periodic scheduling achieves the lowest mean waiting delay as shown by (6). Since one packet is delivered only in one time slot, after waiting, the packet is transmitted within one slot. Therefore, (7) holds for the lower bound of mean packet transmission delay.

As the second moment cannot be easily determined, the formulation of lower bound will be used in the following 
analysis. For simplicity, the superscript "LB" is removed, e.g., using $D_{n}$ instead of $D_{n}^{\mathrm{LB}}$.

\subsubsection{Determination of multichannel superframe}

In the following, we present the determination of multichannel scheduling $\mathrm{A}$. With $R_{n}$ resource blocks allocated to sensor $n$, the mean scheduling interval $\bar{s}_{n}$ is fixed according to (3). From (5), it is shown that a smaller second moment of scheduling interval $s_{n}^{(2)}$ will provide a lower mean waiting delay as well as mean transmission delay in (7). Hence, a good superframe should make second moment of scheduling intervals as small as possible. In this paper, with the consideration of fairness among sensors, we try to find the optimal schedule A to satisfy

$$
\mathbf{A}^{*}=\arg \min _{n \in[1, N]} \max _{n} s_{n}^{(2)}
$$

Problem (8) is an integer programming problem, which normally does not have an analytical solution. To this end, we design a greedy multichannel superframe determination (GMSD) algorithm as shown in Algorithm 1.

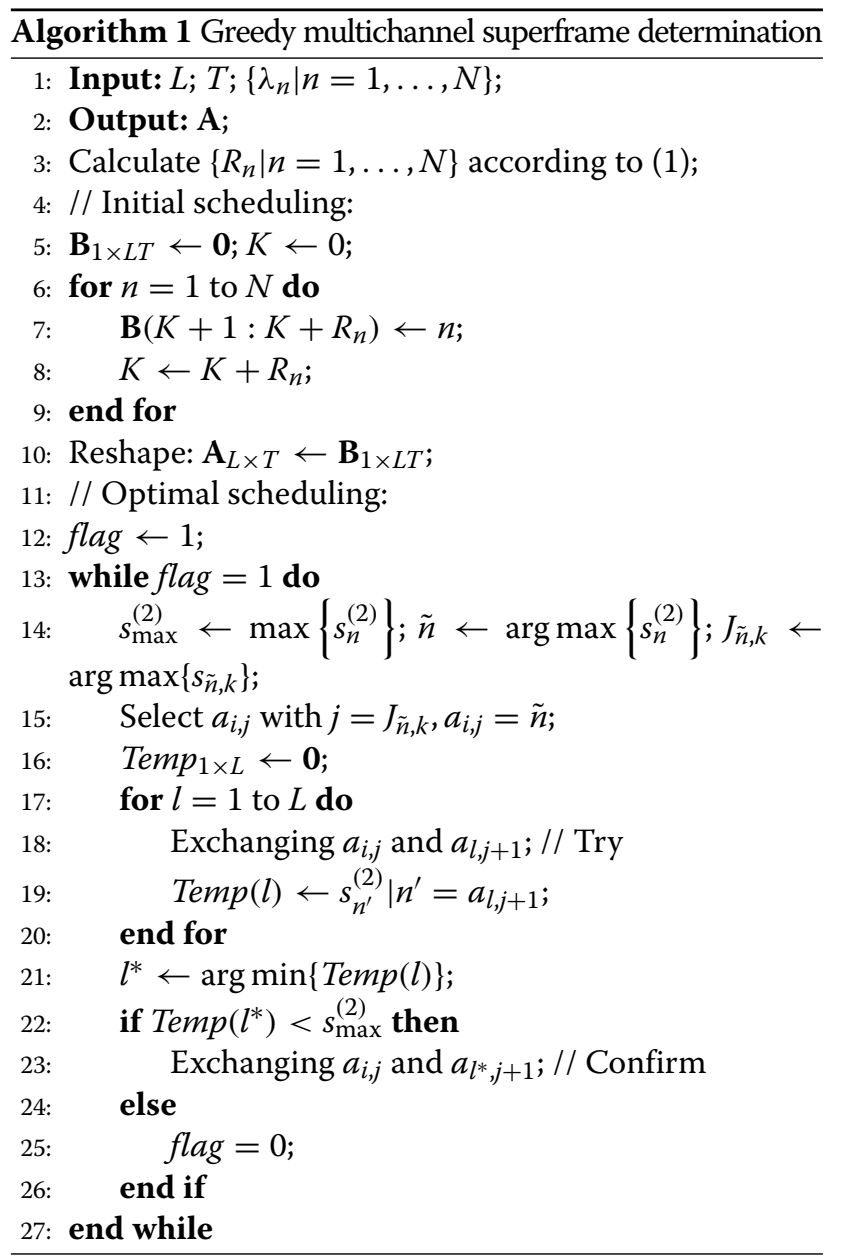

The GMSD algorithm is conducted in two phases. The first phase is initial scheduling. The resource blocks $\left\{R_{n}\right\}$ are sequentially distributed to a one-dimensional sequence, i.e., $\mathbf{B}_{1 \times L T}$ in Algorithm 1. Then, reshape $\mathbf{B}$ to A.

The second phase is optimal scheduling in a greedy manner. It tries to decrease maximal second moment of scheduling intervals iteratively. In each iteration, the sensor $\tilde{n}$ with maximal second moment $s_{\max }^{(2)}$ is first selected. Then, find the largest scheduling interval of $\tilde{n}$ and its left schedule $a_{i, j}$. Try to exchange $a_{i, j}$ with its neighboring schedules $\left\{a_{l, j+1} \mid l=1, \ldots, L\right\}$ and record the minimal second moment from the sensors $\left\{n^{\prime} \mid n^{\prime}=a_{l, j+1}, l=\right.$ $1, \ldots, L\}$. If this minimal second moment obtained by sensor $n^{\prime}=a_{l^{*}, j+1}$ is smaller than $s_{\max }^{(2)}$, confirm the scheduling exchange between $\tilde{n}$ and $n^{\prime}$; otherwise, cancel the exchange and end the scheduling.

\subsection{Separate design principle for priority-aware packet collection}

Based on the multichannel superframe design in previous subsection, the SDP is obtained in this subsection.

Figure 1 gives the illustration of SDP by an instance with three priority classes and two wireless channels. The notation " $n_{c}$ " in the first sub-figure represents that the corresponding slot on the specific channel is scheduled to the sensor $n$ from priority class $c$. As shown in the figure, SDP separately schedules the transmission for each class of sensors with the multichannel superframe, e.g., three superframes indicated by different color depths for three classes of sensors. With TDMA scheduling, the collisions among the sensors in the same priority class are avoided. However, as each resource block is scheduled to sensors of different priority, it may lead to collisions when sensors of different priorities transmit packet simultaneously. As a solution, SDP introduces a priority-aware transmission coordination mechanism by introducing the priority indicator, which coordinates different sensors to transmit packet in a given priority order. The details of SDP are presented by the following two phases.

1. Separate scheduling: Based on the multichannel superframe presented in Section 4.1.1, we devise the superframe for each class of sensors separately. For the superframe of period $T$ slots on $L$ channels, the number of resource blocks allocated to each sensor in priority class $c$ is

$$
R_{n, c}=\frac{\lambda_{n, c}}{U_{c}} L T, n=1, \ldots, N_{c}, c=1, \ldots, C,
$$

where $U_{c}=\sum_{n=1}^{N_{c}} \lambda_{n, c}$ is the total packet rate of sensors in priority class $c$. Note that there are two special cases due to that each sensor work on a single channel. The first case is that the number of sensors is smaller than the number of 


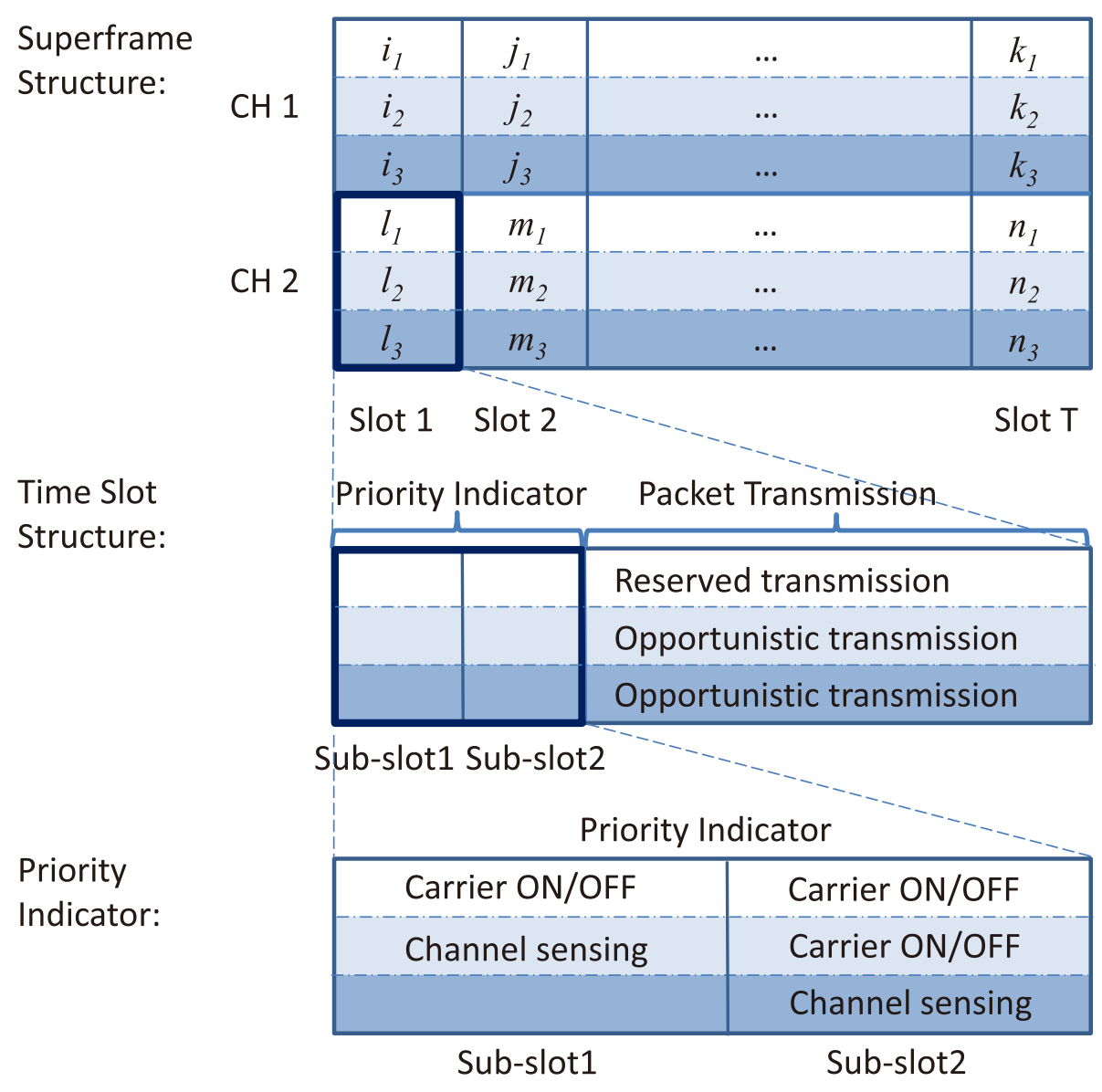

Priority 1

Priority 2

Priority 3

Fig. 1 An instance of SDP-based multichannel scheduling for priority-aware packet collection: three priority classes and two channels

channels. In this case, each sensor is assigned to one channel. The other case is that one sensor may be allocated more than $T$ slots according to its proportional coefficient. In this case, the sensor should be allocated $T$ slots and the remaining slots will be shared by others. Here, we focus on the analysis of the general cases. The two special cases can be analyzed in the similar way.

For realization, we use the floor and ceil functions to make sure that $R_{n, c}$ is an integer. Then, the multichannel superframes $\mathbf{A}_{c}$ for each priority class of sensors can be devised by GMSD algorithm separately and independently.

2. Priority-aware transmission coordination mechanism: Since the transmission scheduling of each class of sensors is designed by fully utilizing the resource blocks in the superframe, a priority-aware transmission coordination mechanism is required to resolve the collisions among the sensors with different priorities. As shown in Fig. 1, we introduce a priority indicator by setting $C-1$ sub-slots at the beginning of each time slot. The time length of a sub-slot can be set to the backoff period defined in IEEE 802.15.4 protocol. For the priority class $c=1$, its sensors have the highest transmission priority. Hence, if one sensor of class 1 has data packet to transmit, it first transmits the carrier signal during the $C-1$ sub-slots as the priority indicator and then transmit the data packet during the remaining part of the slot. For any priority class $c>1$, its sensors have to transmit data packet opportunistically. If one sensor from priority class $c$ has data packet to transmit, it has to sense the channel for only one subslot, i.e., the sub-slot $c-1$. If the channel is sensed idle during this sub-slot, it transmits the carrier signal during the remaining sub-slots $c, \ldots, C-1$ and then transmits the data packet; otherwise, it postpones the channel access try to the next scheduled slot. Note that more complicated preamble signal can be used instead of the carrier signal, thus to embed the SDP into some existing communication protocols. 


\section{Performance analysis}

\subsection{Mean transmission delay analysis based on SDP}

Consider the packet transmission process of each sensor as a queueing process. The transmission delay of each sensor is not only affected by the first and second moments of scheduling intervals but also affected by the transmission priority coordination in SDP. Note that if the packet arrival rates satisfy $\sum_{c=1}^{C} \sum_{n=1}^{N_{c}} \lambda_{n, c}<L$, the queueing processes of all sensors are stable with the proportional resource allocation by (9); thus, the packet transmission in the network is stable. In the steady state, the probability of the scheduled resource block used by sensor $n$ from priority class $c$ is the product of this sensor's packet rate and its mean scheduling interval, i.e.,

$$
\rho_{n, c}=\lambda_{n, c} \bar{s}_{n, c}=\lambda_{n, c} \frac{T}{R_{n, c}}=\frac{U_{c}}{L}=\rho_{c},
$$

where $\bar{s}_{n, c}$ is the mean scheduling interval of sensor $n$ from priority class $c$ and $\rho_{c}$ is the ratio of used network capacity by class $c$. According to the queueing theory, $\rho_{c}$ is also called utilization. Equation (10) implies that each resource block that is used by one sensor from priority class $c$ is equivalent to the utilization of the priority class $c$, i.e., $\rho_{c}$.

Recall the priority-aware transmission coordination mechanism. For the highest priority $c=1$, the sensors have the absolute access right to their scheduled resource blocks. That means if one sensor from priority class 1 has packets to transmit during the scheduled slot, it can access to the scheduled channel during the scheduled slot with probability 1 , denoted by $p_{1}=1$ here. However, the occupation ratio of the resource by this sensor from priority class 1 is $\rho_{1}$, i.e., the utilization of priority class 1 . For the sensors from priority class 2 , they have lower access right than the sensors from priority class 1 , which means that the sensors from priority class 2 can access to their scheduled resource block with probability $p_{2}=1-\rho_{1}$. In general, for sensors from priority class $c$, the occupation ratio of their scheduled resource blocks by the higher priority sensors is $\sum_{i=1}^{c-1} \rho_{i}$; thus, the probability that they can access to the scheduled resource blocks is

$$
p_{c}=1-\sum_{i=1}^{c-1} \rho_{i}, \quad c=1, \ldots, C \text {. }
$$

With the priority-aware transmission coordination mechanism, the mean transmission interval of the sensors from priority class $c$ is equivalent to

$$
{\overline{s^{\prime}}}_{n, c}=\frac{\bar{s}_{n, c}}{p_{c}},
$$

and the lower bound of the second moment of transmission interval is

$$
s_{n, c}^{\prime}(2)=\left(\frac{\bar{s}_{n, c}}{p_{c}}\right)^{2} \text {. }
$$

As a result, the lower bound of mean waiting delay of packet transmission for sensor $n$ from priority class $c$ can be obtained as

$$
W_{n, c}=\frac{\lambda_{n, c} s^{\prime}{ }_{n, c}^{(2)}}{2\left(1-\lambda_{n, c}{\overline{s^{\prime}}}_{n, c}\right)}=\frac{\rho_{c}{ }^{2}}{2 \lambda_{n, c}\left(1-\sum_{i=1}^{c-1} \rho_{i}\right)\left(1-\sum_{i=1}^{c} \rho_{i}\right)} .
$$

Since the successful transmission probability of a sensor from priority class $c$ is $p_{c}$, the mean time slots from the slot for this packet to be transmitted to the slot at which it is successfully transmitted can be given by

$$
\Delta_{n, c}=p_{c} \cdot 1+\sum_{k=1}^{\infty}\left(1-p_{c}\right)^{k} p_{c}\left(1+k \bar{s}_{n, c}\right)=\frac{\rho_{c} \sum_{i=1}^{c-1} \rho_{i}}{\lambda_{n, c}\left(1-\sum_{i=1}^{c-1} \rho_{i}\right)}+1
$$

Hence, the lower bound of the mean packet transmission delay of sensor $n$ from priority class $c$ is

$$
\begin{aligned}
D_{n, c}= & \frac{\rho_{c}^{2}}{2 \lambda_{n, c}\left(1-\sum_{i=1}^{c-1} \rho_{i}\right)\left(1-\sum_{i=1}^{c} \rho_{i}\right)} \\
& +\frac{\rho_{c} \sum_{i=1}^{c-1} \rho_{i}}{\lambda_{n, c}\left(1-\sum_{i=1}^{c-1} \rho_{i}\right)}+1 .
\end{aligned}
$$

\subsection{Comparison with non-overlap scheduling}

For general TDMA-based superframe design, in order to avoid the collision, the resource block allocation is exclusive, such as GTS allocation in IEEE 802.15.4e and superframe design in WirelessHART. We call this type of scheduling as non-overlap scheduling. In this subsection, we analyze the transmission delay performance of nonoverlap scheduling and compare it to delay performance of the SDP-based scheduling.

For non-overlap scheduling, each resource block of the superframe is scheduled to one sensor exclusively. In other words, the resource blocks of the superframe cannot be fully scheduled to one priority class. Denote $\left\{\alpha_{c} \mid c=\right.$ $1, \cdots, C\}$ as the weight coefficients of resource allocation to each priority class of sensors, where $\sum_{c=1}^{C} \alpha_{c}=1$. With proportional resource allocation in (1), the number of resource blocks allocated to each sensor in priority class $c$ is

$$
\widetilde{R}_{n, c}=\frac{\lambda_{n, c}}{U_{c}} \alpha_{c} L T, n=1, \ldots, N_{c}, c=1, \ldots, C .
$$

where the tilde symbol is used to differ from the metric notations of SDP. Accordingly, the mean transmission interval of sensor $n$ from priority class $c$ is

$$
\widetilde{s}_{n, c}=\frac{\rho_{c}}{\lambda_{n, c} \alpha_{c}}
$$


and its corresponding lower bound of the second moment is

$$
\widetilde{s}_{n, c}^{(2)}=\left(\widetilde{s}_{n, c}\right)^{2} .
$$

As a result, the lower bound of the mean waiting delay of sensor $n$ from priority class $c$ can be obtained as

$$
\widetilde{W}_{n, c}=\frac{\rho_{c}^{2}}{2 \lambda_{n, c} \alpha_{c}\left(\alpha_{c}-\rho_{c}\right)},
$$

and the lower bound of the transmission delay is

$$
\widetilde{D}_{n, c}=\frac{\rho_{c}^{2}}{2 \lambda_{n, c} \alpha_{c}\left(\alpha_{c}-\rho_{c}\right)}+1 .
$$

Based on the above analysis, we have the following theorem to demonstrate the advantage of SDP.

Theorem 1. For priority-ware packet collection, SDPbased scheduling achieves lower mean waiting delay for each priority class than non-overlap scheduling.

Proof. Considering the stability of packet transmission in non-overlap scheduling, it is easy to drive that $\rho_{c}<$ $\alpha_{c}<\left(1-\sum_{i \in[1, C], i \neq c} \rho_{i}\right)$. From (20), a larger $\alpha_{c}$ results in a smaller mean waiting delay. However, for $\forall c \in[1, C]$, we have

$$
1-\sum_{i \in[1, C], i \neq c} \rho_{i} \leq 1-\sum_{i=1}^{c-1} \rho_{i},
$$

where the equality holds only when $c=C$, i.e., the lowest priority class. As $\alpha_{c}<\left(1-\sum_{i \in[1, C], i \neq c} \rho_{i}\right)$, we can conclude that $W_{n, c}<\widetilde{W}_{n, c}$ for $\forall c \in[1, C]$. Thus, Theorem 1 holds.

Remark 1. Based on non-overlay scheduling, only the sensor in the lowest priority $C$ can possibly acquire the same waiting delay as the SDP-based scheduling does. However, in such case, the other priority classes are only allocated with resource at a portion of $\alpha_{c}=\rho_{c}, c=$ $1, \cdots, C-1$. That means the waiting delay of these classes of sensors will approach infinity. Although we theoretically have $\widetilde{D}_{n, c}<D_{n, c}$ for certain class $c$ given large $\alpha_{c}$, it will lead to rapid increase of the transmission delay of other classes. More deepgoing evaluations will be presented by performance evaluation in the following section.

\section{Simulation study and experiments}

In this section, we first evaluate the mean packet transmission delay of sensors for SDP-based multichannel scheduling. The comparison of simulated results and theoretical lower bounds is presented. Then, we provide the comparisons between SDP-based and IEEE 802.15.4e-based multichannel scheduling. To show the feasibility of the SDP, the experiments on the SDR platform are also conducted.

The network parameters for simulations are set as shown in Table 1. The packet rates of sensors are randomly initialized but keep the utilization as shown in the table. As IEEE 802.15.4e support multi-superframe, here, we set the period of multi-superframe as $16 \times 2$ slot whereby the single superframe length is 16 slots.

\subsection{Mean packet transmission delay evaluation}

This part shows the simulation results of mean packet transmission delay of SDP-based multichannel scheduling and its corresponding theoretical lower bound, which are marked by "SDP" and "SDP Theo," respectively. To demonstrate the influence of second moment of scheduling interval, we also conduct the simulation based on sequential scheduling (marked by "Seq") with the multichannel superframe obtained by initial scheduling in Algorithm 1 (i.e., A at line 10).

The simulation results are presented in Fig. 2. It is shown that with simple sequential scheduling, the mean packet transmission delay of each priority class is larger than that of the SDP-based scheduling. Using the GMSD, the mean packet transmission delay is effectively decreased and approaches to the theoretical lower bound. Because some sensors, e.g., sensor 3 and sensor 4 in priority class 1 , are allocated exact one channel, respectively, they have the same mean packet transmission delay with different scheduling approaches. Further, we show the average delay of all sensors from same priority class in Fig 3. As expected, the average transmission delay of each priority class increases with its priority order. However, compared to sequential scheduling, the increment of SDP-based scheduling is relatively small. We also note that based on SDP, an increment of sensors brings in a small increment of the delay gap between the simulated result and the theoretical result. That is because for a large number of sensors, it is non-trivial to approach to the lower bound of mean packet transmission delay.

Table 1 Network parameters for simulation

\begin{tabular}{ll}
\hline Number of channels: & $L=16$ \\
Period of superframe: & $T=16 \times 2$ \\
Number of priority classes: & $C=4$ \\
Number of sensors in each priority class: & $N_{1}=20, N_{2}=30$, \\
& $N_{3}=40, N_{4}=50$ \\
Utilization of each priority class: & $\rho_{1}=0.1, \rho_{2}=0.1$, \\
& $\rho_{3}=0.2, \rho_{4}=0.2$ \\
\hline
\end{tabular}



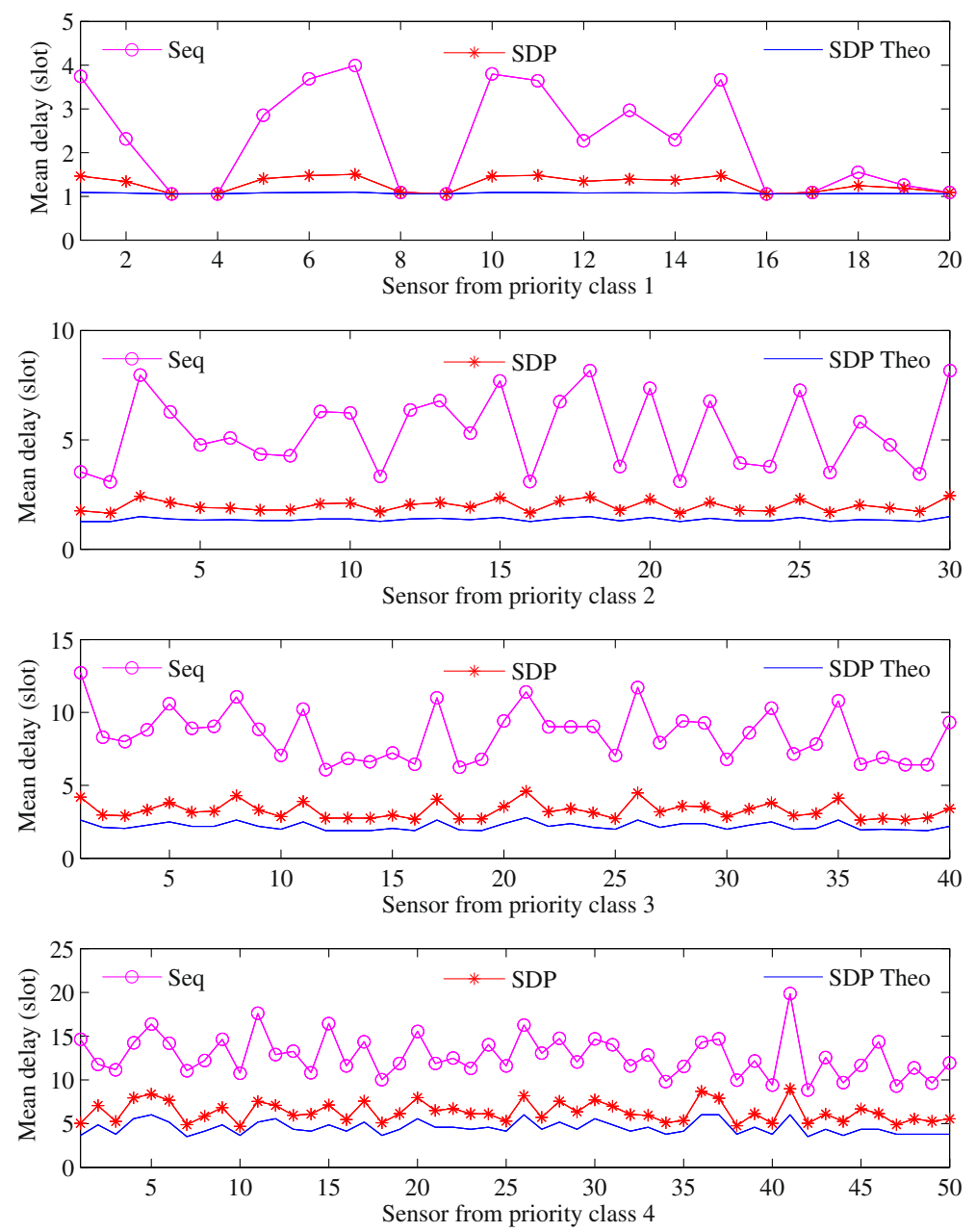

Fig. 2 Mean packet transmission delay of each sensor in four priority classes: SDP vs. sequential scheduling

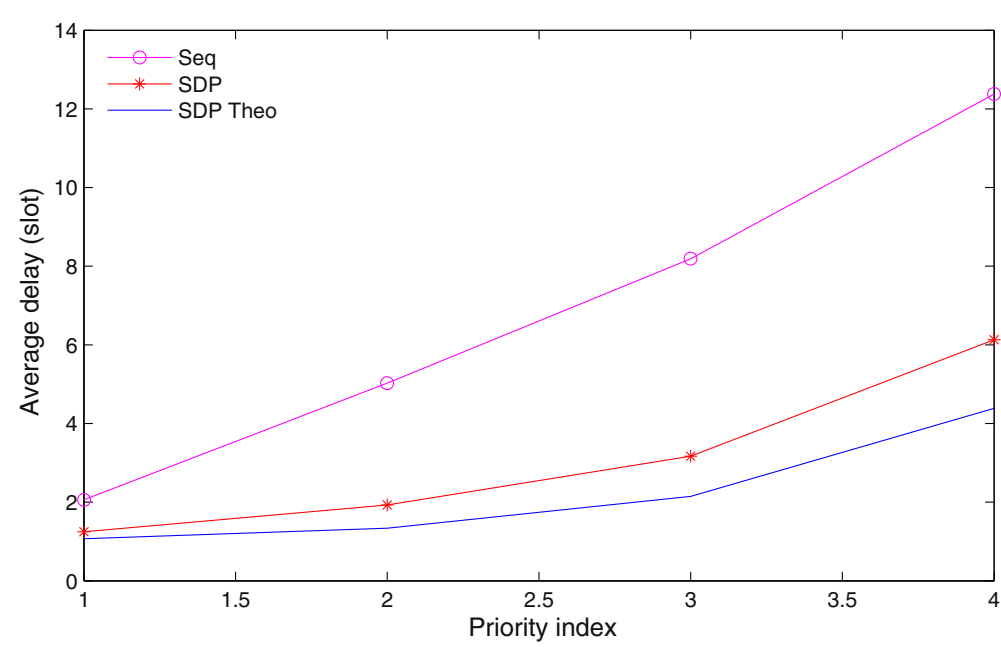

Fig. 3 Average packet transmission delay of each priority class of sensors 


\subsection{SDP vs. non-overlap scheduling}

This part shows the comparison between the SDP-based scheduling and the non-overlap scheduling (marked by "NS"). As illustrated in Section 5.2, the transmission delay of sensors from different priority classes is affected by the weighted coefficients $\left\{\alpha_{c}\right\}$. We first compare SDP to NS with moderate weighted coefficients $\alpha_{1}=0.2, \alpha_{2}=$ $0.2, \alpha_{3}=0.3$, and $\alpha_{4}=0.3$. The results shown in Fig. 4 indicate that SDP achieves much lower mean packet transmission delay of each priority class of sensors than the NS does. The average delays of each class achieved by SDP are only $16,18,23$, and $36 \%$ of the average delays achieved by NS, respectively.

From (16) and (21), the mean packet transmission delay of low-priority class based on NS can theoretically be lower than the one based on SDP by adjusting the weighted coefficients. In the following, we check the case that the weighted coefficient $\alpha_{4}$ varying in its range $\rho_{4} \sim\left(1-\sum_{i=1}^{3} \rho_{i}\right)$, i.e., $0.2 \sim 0.6$. The other weighted coefficients are set as $\alpha_{i}=\left(1-\alpha_{4}\right) \rho_{i} /\left(\sum_{i=1}^{3} \rho_{i}\right)$ for $i=1,2,3$. The results are presented in Fig. 5 . It is shown that the average delays of priority classes 1,2 , and 3 based on SDP are lower than the ones based on NS. As for priority class 4, the average delay based on NS can hardly be lower than that based on SDP. Particularly when $\alpha_{4} \geq 0.55$, the average delay of priority class 4 based on NS is lower; however, the average delays of other high priority classes are dramatically increased ( $>10^{2}$ slots) and which is not acceptable. From simulation results, it can be concluded that the proposed SDP completely outperforms the non-overlap scheduling.

\subsection{SDP vs. IEEE 802.15.4e}

For comparison, we also evaluate the transmission delay with multichannel scheduling method based on IEEE 802.15.4e. As introduced in Section 2, in IEEE 802.15.4e, high-priority sensors are scheduled to transmit in CFP
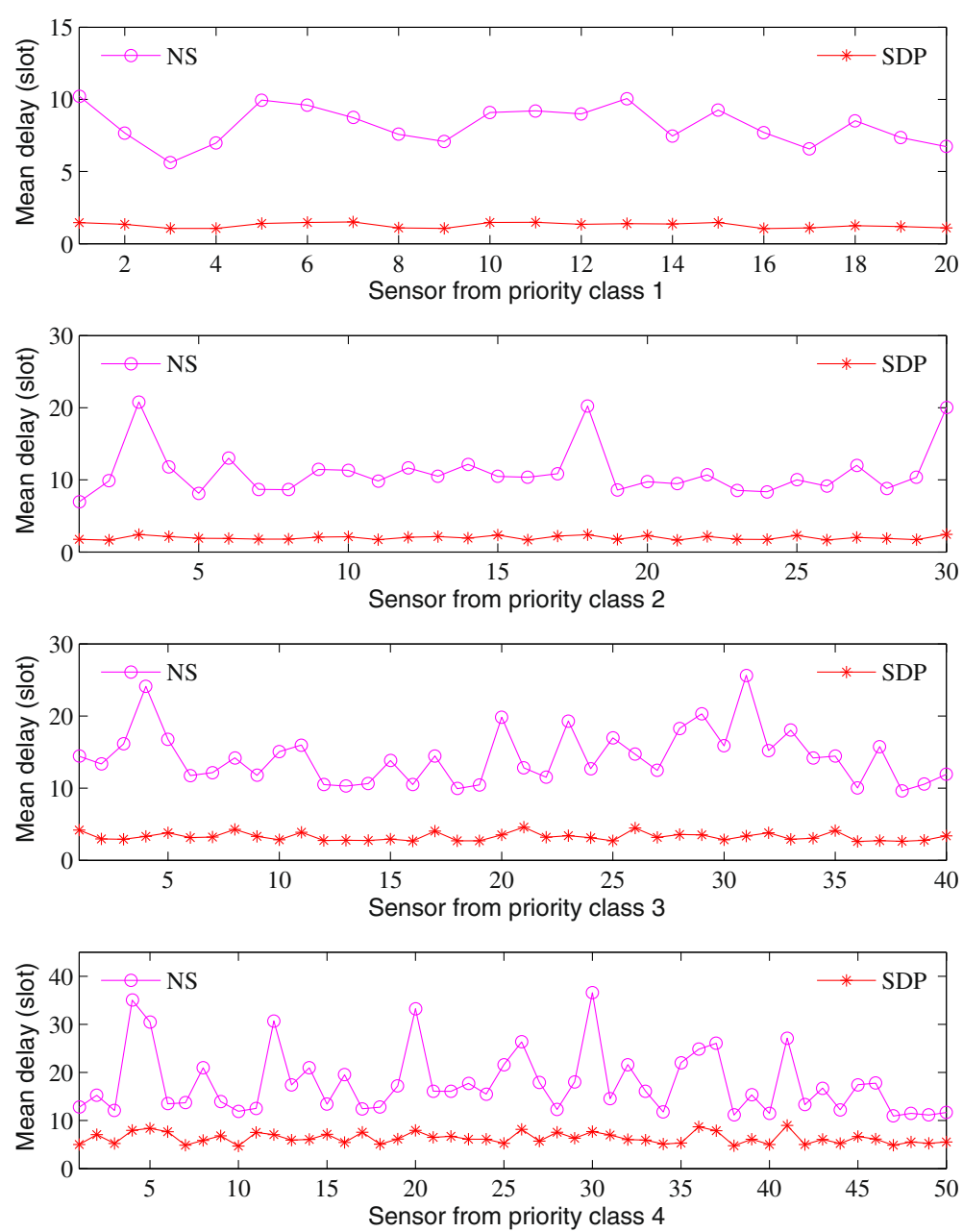

Fig. 4 Mean packet transmission delay of each sensor in four priority classes: SDP vs. non-overlap scheduling 


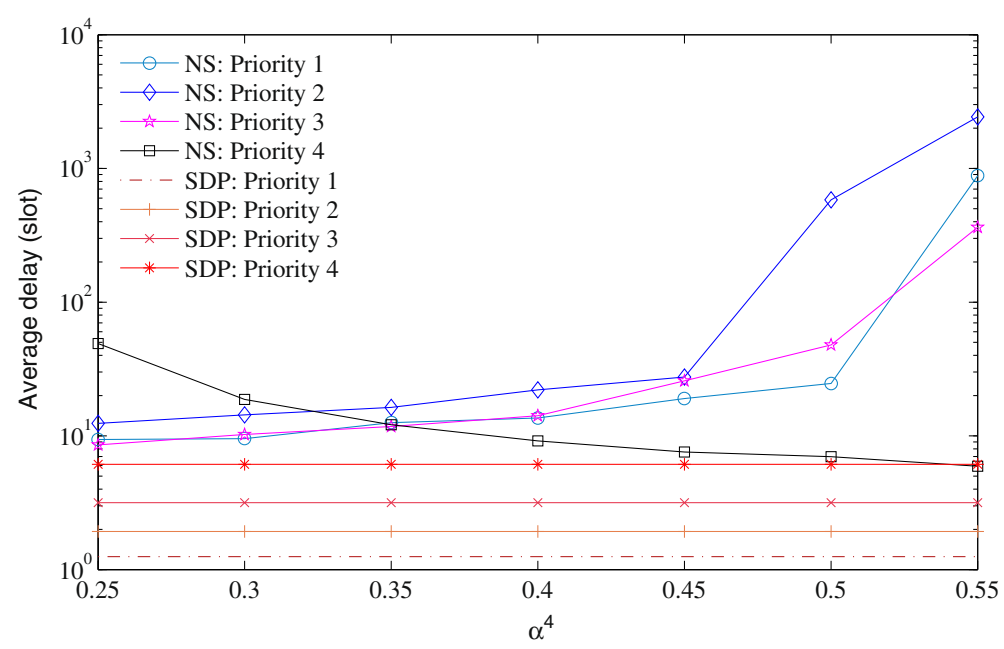

Fig. 5 Average packet transmission delay of each priority class: $\alpha_{4}$ varying from 0.25 to 0.55 in NS

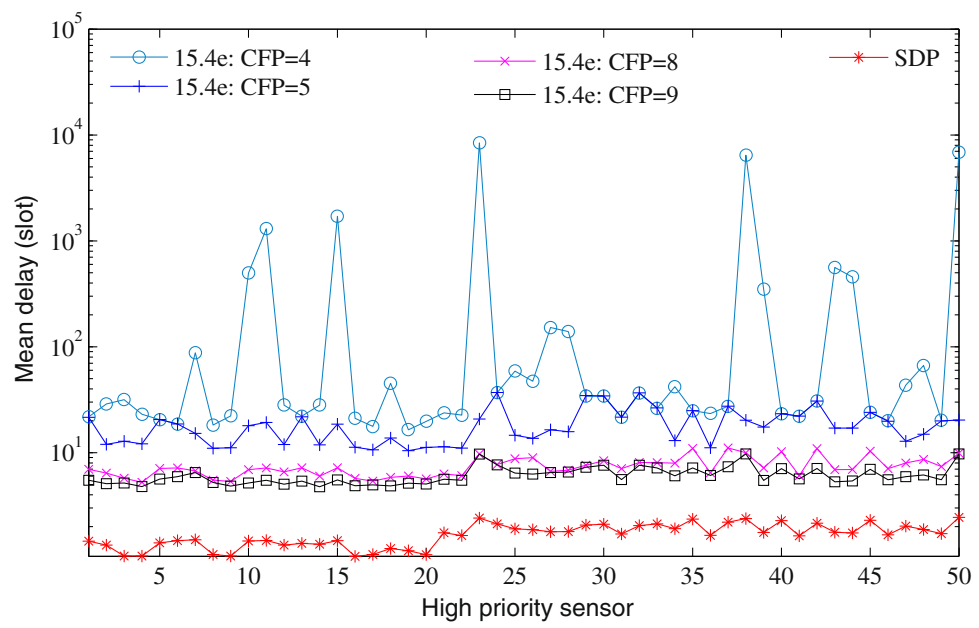

(a) Mean packet transmission delays of high priority sensors

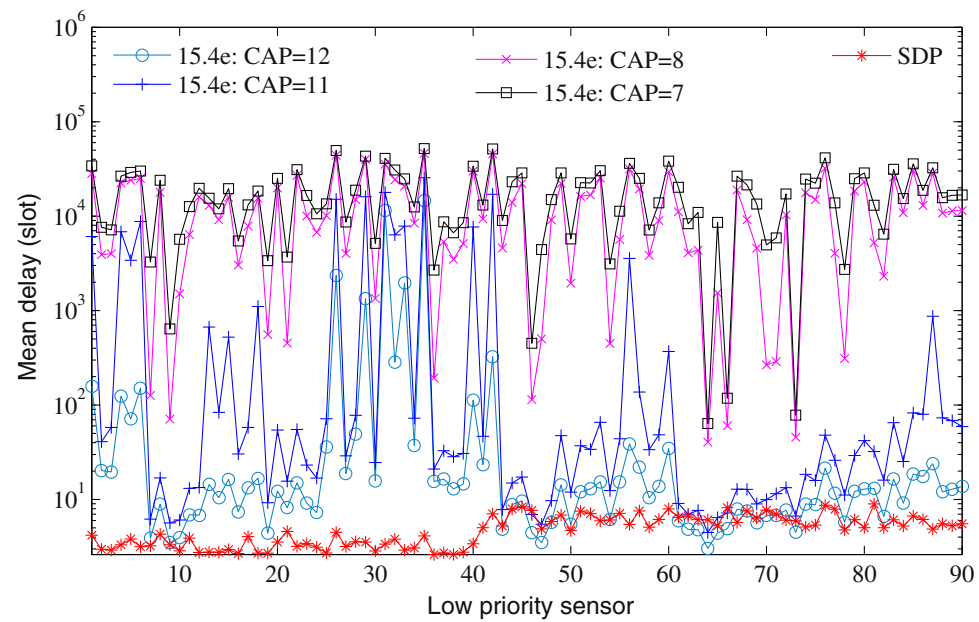

(b) Mean packet transmission delays of low priority sensors

Fig. 6 Comparison results: mean packet transmission delay. a Mean packet transmission delays of high-priority sensors. b Mean packet transmission delays of low-priority sensors 


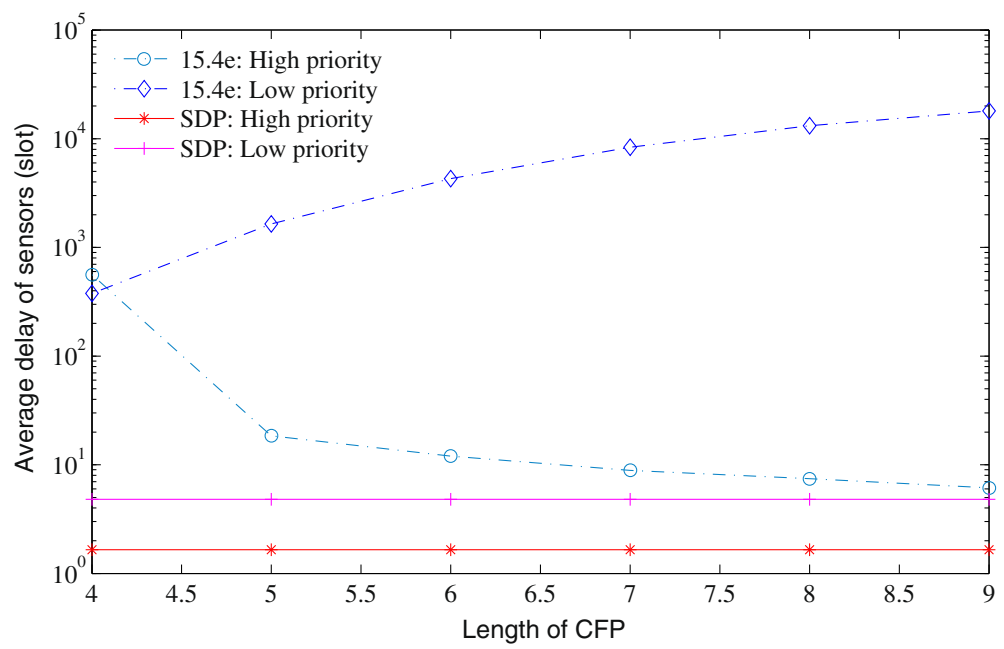

Fig. 7 Comparison results: average delay of all sensors with same priority

with TDMA manner and low-priority sensors are committed to transmit in CAP with CSMA/CA manner. The parameters $N B, a M i n B E, a M a x B E$, and $C W$ for CSMA are set to default values in IEEE 802.15.4. Here, we group the priority classes 1 and 2 into one class, named high-priority class and group priority classes 3 and 4 into one class, named low-priority class. We partition the low-priority sensors into 16 groups and assign each group with one channel, thus to avoid the over crowded on some channels. The CFP and CAP are tunable as used in [14]. To guarantee the stability of packet transmission, under network settings in Table 1 , we have $4 \leq$ CFP $\leq 9$ and $7 \leq \mathrm{CAP} \leq 12$.

Figure 6 compares the mean packet transmission delay between SDP and IEEE 802.15.4e. From Fig. 6a, it can be seen that SDP, with full use of the multichannel superframe, achieves lower mean packet transmission delay for high-priority sensors than IEEE 802.15.4e. Since the superframe of IEEE 802.15.4e has to reserve CAP for lowpriority sensors, the CFP is shorter compared to the one of SDP. From the figure, when the CFP is small, e.g., 4 slots, the mean packet transmission delay becomes very large. The comparison results of the packet transmission of low priority sensors are in Fig. 6b. It is shown that CSMA-based packet transmission results in large packet transmission delay even if the CAP is set relatively long. When the CAP is set small, the packet transmission is almost blocked. The simulation results demonstrate the great advantage of SDP over the CSMA mechanism.

The evaluation of the average delay of the sensors from the same priority class is shown in Fig. 7. From the figure, it clearly shows that both the average delays of highand low-priority sensors achieved by SDP are lower than the ones achieved by IEEE 802.15.4e. In addition, the packet transmission delay performance of IEEE 802.15.4e is even worse than non-overlap TDMA scheduling, for both low- and high-priority classes of sensors. In summary, for priority-aware packet collection in large-scale industrial WSNs, TDMA-based scheduling is better than CSMA-based channel access. The proposed SDP provides the lowest mean packet transmission delay.

\subsection{Experiments on USRP platform}

To demonstrate the feasibility of the proposed SDP scheme, preliminary experiments have been conducted on the SDR platform implemented by USRPs (universal software radio peripherals). As shown in Fig. 8, five USRPs (version: NI USRP2921) are used during the experiments, where one is employed as the AP and the other four are used as the sensor nodes. Due to the limited devices, we consider the scenario where two high-priority sensors and two low-priority sensors try to transmit the packets to one AP on one channel. The packet rates are set to 0.08 and 0.12 packets per slot for two high-priority sensors and 0.10 and 0.20 packets per slot for two low-priority

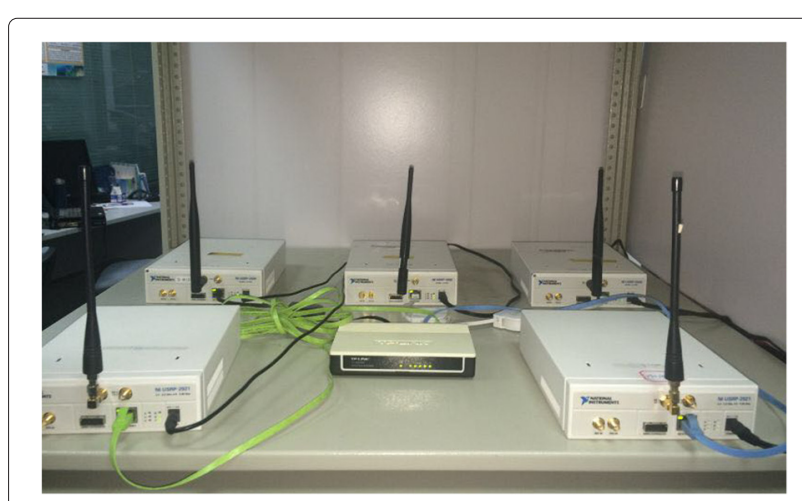

Fig. 8 SDR platform implemented by USRPS 


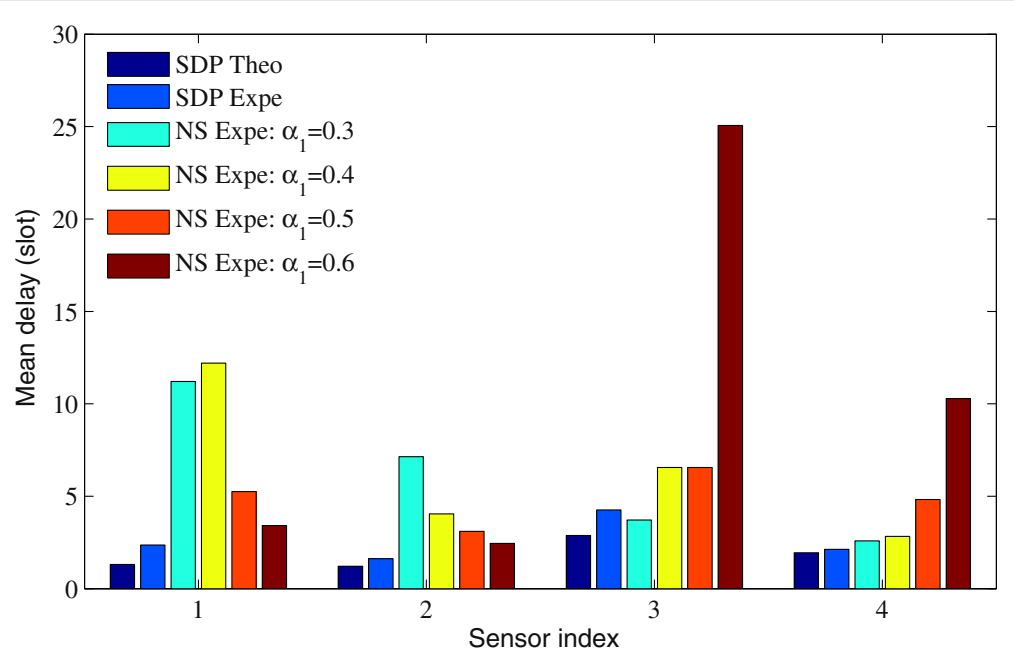

Fig. 9 Experiments results: mean packet transmission delay

sensors, respectively. As defined in IEEE 802.15.4, the OQPSK modulation/demodulation is used, the data rate is set to $250 \mathrm{Kbps}$ and the bandwidth is $2 \mathrm{MHz}$. To avoid the interference from WiFi signal, the central frequency of the channel is set to $2425 \mathrm{MHz}$. The transmitting power of the URSP is $-10 \mathrm{dBm}$. Constrained to the processing speed of the USRP, the time slot is set to $100 \mathrm{~ms}$.

On this SDR platform, the experiments for SDP- and NS-based scheduling are conducted. Figure 9 presents the experimental results after running 10,000 time slots for each experiment.

First, it is shown that the experimental results of the mean packet transmission delay for each sensor based on SDP (denoted by "SDP Expe" in the figure) is close to the theoretical lower bound. During the experiment, two reasons make them slightly larger than the lower bound: (1) the scheduling based on superframe design is not strictly periodic, which will increase the mean delay as proved by (5) in Section 4.1.2 and (2) the packet loss will occur caused by the interference, and the retransmission increases the transmission delay.

The comparison with the non-overlap scheduling (denoted by "NS Expe" in the figure) is also presented in Fig. 9. Let the weighted coefficients $\alpha_{1}$ for high-priority sensors varies from 0.3 to 0.6 , and $\alpha_{2}$ for low priority sensors is set to $\alpha_{2}=1-\alpha_{1}$. Not surprisingly, the results demonstrate that the SDP outperforms the non-overlap scheduling. It is shown when $\alpha_{1}=0.3$, more resource is allocated to the lower priority sensors. As a result, sensor 3 gains a lower delay based on NS than that based on SDP; however, in this case, the delay of high-priority sensors are even higher than the delay of low-priority sensors which is not reasonable in application. When more resource is allocated to high-priority sensors, i.e., $\alpha_{1}>0.3$, it is shown that SDP achieves the lower mean transmission delay than non-overlap scheduling for all of the four sensors.

\section{Conclusions}

In this paper, a separate design principle-based multichannel scheduling scheme is proposed for priorityaware packet collection in ICPS. The greedy multichannel scheduling determination algorithm is developed to optimize the design of the multichannel superframe. A priority-aware packet transmission coordination mechanism is devised to solve the channel access collision. Simulation results are provided to demonstrate that the SDP-based multichannel scheduling achieves lower mean transmission delay of each priority class than that achieved by non-overlap scheduling and IEEE 802.15.4ebased scheduling. The feasibility of the proposed SDP is demonstrated through some preliminary experimental results. In future work, we will consider the extension of SDP to a large-scale network topology for ICPS, such as cluter-tree topology.

\section{Competing interests}

The authors declare that they have no competing interests.

\section{Acknowledgements}

This work was supported in part by NSF of China under the grants 61221003 , U1405251, 61290322, 61371085, 61431008, and 61273181, in part by National High Technology Research and Development Program of China (863 Program) under 2015AA01A702, in part by Ministry of Education of China under NCET-13-0358, and in part by Science and Technology Commission of Shanghai Municipality (STCSM), China under 13QA1401900.

\section{Author details}

${ }^{1}$ Department of Automation, Shanghai Jiao Tong University, 800, Dongchuan Rd., Minhang District, 200240 Shanghai, People's Republic of China. ${ }^{2}$ Key Laboratory of Systems Control and Information Processing, Ministry of Education of China, 800, Dongchuan Rd., Minhang District, 200240 Shanghai, People's Republic of China. ${ }^{3}$ School of Information Security Engineering, Shanghai Jiao Tong University, 800, Dongchuan Rd., Minhang District, 200240 Shanghai, People's Republic of China. 
Received: 20 November 2015 Accepted: 24 February 2016 Published online: 02 March 2016

\section{References}

1. F Xia, A Vinel, R Gao, L Wang, T Qiu, Evaluating IEEE 802.15.4 for cyber-physical systems. EURASIP J. Wireless Commun. Netw. 2011(596397), 1-14 (2011)

2. K-D Kim, PR Kumar, Cyber physical systems: a perspective at the centennial. Proc. IEEE. 100(Special Centennial Issue), 1287-1308 (2012)

3. J Lee, B Bagheri, H-A Kao, A cyber-physical systems architecture for industry 4.0-based manufacturing systems. Manuf. Lett. 3, 18-23 (2015)

4. PC Evans, M Annunziata, Industrial Internet: Pushing the Boundaries of Minds and Machines. (General Electric Co., Schenectady, New York, 2012)

5. A Willig, E Uhlemann, Deadline-aware scheduling of cooperative relayers in TDMA-based wireless industrial networks. Wireless Netw. 20(1), 73-88 (2014)

6. IEEE 802.15.4, Part 15.4: wireless medium access control (MAC) and physical layer (PHY) specifications for low-rate wireless personal area networks (LR-WPANs). IEEE Standard for information technology (2006)

7. C Wang, T Jiang, Q Zhang, ZigBee ${ }^{\circledast}$ Network Protocols and applications. (CRC Press, Boca Raton, Florida, 2014)

8. IEC Std. 62,591, Industrial communication networks - wireless communication network and communication profiles-WirelessHART. Int. Electrotech. Commission (2010)

9. ISA100.11a, Wireless systems for industrial automation: process control and related applications. ISA100 Standards Committee (2009)

10. IEC Std. 62,061, Industrial communication networks - fieldbus specifications-WIA-PA communication networks and communication profiles. Int. Electrotech. Commission (2011)

11. G Anastasi, M Conti, M Di Francesco, A comprehensive analysis of the MAC unreliability problem in IEEE 802.15. 4 wireless sensor networks. IEEE Trans. Ind. Inform. 7(1), 52-65 (2011)

12. IEEE 802.15.4e, IEEE standard for local and metropolitan area networks - part. 15.4: low-rate wireless personal area networks (LR-WPANs) —amendament1: MAC sublayer. IEEE Standard for information technology (2012)

13. F Lin, C Chen, C Hua, X Guan, in Proc. 10th International Conference on Wireless Algorithms, Systems and Applications (WASA'15). SDP: separate design principle for multichannel scheduling in priority-aware packet collection (Springer, Qufu, Shandong, China, 2015), pp. 356-365

14. MHS Gilani, I Sarrafi, M Abbaspour, An adaptive CSMA/TDMA hybrid MAC for energy and throughput improvement of wireless sensor networks. Ad Hoc Netw. 11(4), 1297-1304 (2013)

15. W Shen, T Zhang, F Barac, M Gidlund, PriorityMAC: a priority-enhanced MAC protocol for critical traffic in industrial wireless sensor and actuator networks. IEEE Trans. Ind. Inform. 10(1), 824-835 (2014)

16. A Zhou, M Liu, Z Li, E Dutkiewicz, Cross-layer design for proportional delay differentiation and network utility maximization in multi-hop wireless networks. IEEE Trans. Wireless Commun. 11(4), 1446-1455 (2012)

17. U Bodin, K Wolosz, Proportional throughput differentiation with cognitive load-control on WSN channels. EURASIP J. Wireless Commun. Netw. 2015(1), 1-14 (2015)

18. J Liu, X Jiang, H Nishiyama, N Kato, X Shen, in Proc. 2012 IEEE Wireless Communications and Networking Conference (WCNC'12). End-to-end delay in mobile ad hoc networks with generalized transmission range and limited packet redundancy (IEEE, Shanghai, China, 2012), pp. 1731-1736

19. J Liu, X Jiang, H Nishiyama, N Kato, Generalized two-hop relay for flexible delay control in MANETs. IEEE/ACM Trans. Netw. 20(6), 1950-1963 (2012)

20. J Liu, X Jiang, H Nishiyama, N Kato, On the delivery probability of two-hop relay MANETs with erasure coding. IEEE Trans. Commun. 61(4), 1314-1326 (2013)

21. E Toscano, L Lo Bello, Multichannel superframe scheduling for IEEE 802.15. 4 industrial wireless sensor networks. IEEE Trans. Ind. Inform. 8(2), 337-350 (2012)

22. A Saifullah, Y Xu, C Lu, Y Chen, End-to-end communication delay analysis in industrial wireless networks. IEEE Trans. Comput. 64(5), 1361-1374 (2015)
23. A Azarfar, J-F Frigon, Sansò, Priority queueing models for cognitive radio networks with traffic differentiation. EURASIP J. Wireless Commun. Netw. 2014(1), 1-21 (2013)

24. N Zhang, H Liang, N Cheng, Y Tang, JW Mark, XS Shen, Dynamic spectrum access in multi-channel cognitive radio networks. IEEE J. Selected Areas Commun. 32(11), 2053-2064 (2014)

25. SM Ross, Introduction to Probability Models. (Academic Press, Waltham, Massachusetts, 2014)

\section{Submit your manuscript to a SpringerOpen ${ }^{\circ}$ journal and benefit from:}

- Convenient online submission

- Rigorous peer review

- Immediate publication on acceptance

Open access: articles freely available online

- High visibility within the field

- Retaining the copyright to your article

Submit your next manuscript at $>$ springeropen.com 